\title{
STARTING SOLUTIONS FOR SOME SIMPLE OSCILLATING MOTIONS OF SECOND-GRADE FLUIDS
}

\author{
C. FETECAU AND CORINA FETECAU \\ Received 2 February 2006; Accepted 11 July 2006
}

The exact starting solutions corresponding to the motions of a second-grade fluid, due to the cosine and sine oscillations of an infinite edge and of an infinite duct of rectangular cross-section as well as those induced by an oscillating pressure gradient in such a duct, are determined by means of the double Fourier sine transforms. These solutions, presented as sum of the steady-state and transient solutions, satisfy both the governing equations and all associate initial and boundary conditions. In the special case when $\alpha_{1} \rightarrow 0$, they reduce to those for a Navier-Stokes fluid.

Copyright ( 2006 C. Fetecau and C. Fetecau. This is an open access article distributed under the Creative Commons Attribution License, which permits unrestricted use, distribution, and reproduction in any medium, provided the original work is properly cited.

\section{Introduction}

The flow of a fluid induced by the oscillating shearing motion of a wall is found in many engineering applications such as flows in vibrating media. This problem is termed as Stokes' second problem by Schlichting [1] if the fluid is bounded only by the moving wall. However, it is termed as Couette flow if the fluid is bounded by two parallel walls. The starting solutions of the Stokes problem for a viscous fluid caused by the cosine and sine oscillations of a flat plate have been studied on depth by Erdoğan [2]. These solutions have been recently extended to second-grade fluids by Fetecau and Fetecau [3]. Such motions are not only of fundamental theoretical interest but they also occur in many applied problems such as acoustic streaming around an oscillating body.

The first exact solutions for the flows of a second-grade fluid due to a rigid plate oscillating in its own plane and an oscillating pressure gradient seem to be those of Rajagopal [4]. He has also studied the problem of an infinite rod undergoing torsional and longitudinal oscillations in an incompressible second-grade fluid [5]. His simple but elegant solutions have been later extended to a larger class of non-Newtonian fluids by Rajagopal and Bhatnagar [6]. Similar solutions for different oscillating motions of non-Newtonian 
fluids have been also obtained by Hayat et al. [7-9], Siddiqui et al. [10], and Asghar et al. [11]. Starting solutions for the motion of a second-grade fluid due to the longitudinal and torsional oscillations of an infinite circular cylinder have been recently obtained in [12].

The main purpose of this note is to establish starting solutions corresponding to the motions of a second-grade fluid due to an oscillating edge and an oscillating channel of rectangular cross-section. These solutions are presented as sum of the steady-state and transient solutions and describe the motion of the fluid at small and large times after its initiation. For large times, they tend to the steady-state solutions which are independent of the initial conditions and periodic in time. Finally, for completeness, the exact solutions for the motion of a second-grade fluid due to an oscillating pressure gradient in a duct of rectangular cross-section are also presented. Exact solutions for some motions of second-grade fluids in such domains have been recently obtained by Erdoğan and İmrak [13].

\section{Governing equation}

The Cauchy stress $\mathbf{T}$ in an incompressible homogeneous fluid of second-grade is related to the fluid motion in the following manner [3-5]:

$$
\mathbf{T}=-p \mathbf{I}+\mathbf{S}, \quad \mathbf{S}=\mu \mathbf{A}_{1}+\alpha_{1} \mathbf{A}_{2}+\alpha_{2} \mathbf{A}_{1}^{2}
$$

where $-p$ I denotes the indeterminate spherical stress due to the constraint of incompressibility, $\boldsymbol{S}$ is the extra-stress tensor, $\mu$ is the dynamic viscosity, $\alpha_{1}$ and $\alpha_{2}$ are the normal stress moduli, while the kinematical tensors $\mathbf{A}_{1}$ and $\mathbf{A}_{2}$ are defined through

$$
\mathbf{A}_{1}=(\operatorname{grad} \mathbf{v})+(\operatorname{grad} \mathbf{v})^{T}, \quad \mathbf{A}_{2}=\frac{d \mathbf{A}_{1}}{d t}+\mathbf{A}_{1}(\operatorname{grad} \mathbf{v})+(\operatorname{grad} \mathbf{v})^{T} \mathbf{A}_{1} .
$$

In the above equation, $\mathbf{v}$ denotes the velocity, grad denotes the gradient operator, and $(d / d t)$ denotes the material time differentiation. Of course, all motions are restricted to be isochoric, so that $\mathbf{A}_{1}$ is traceless.

Dunn and Fosdick [14] studied the thermodynamics of fluids of this type and showed that material moduli must satisfy

$$
\mu \geq 0, \quad \alpha_{1} \geq 0, \quad \alpha_{1}+\alpha_{2}=0
$$

The sign of the material moduli $\alpha_{1}$ and $\alpha_{2}$ is the subject of much controversy. A comprehensive discussion on the restrictions for $\mu, \alpha_{1}$, and $\alpha_{2}$ as well as a critical review can be found in the work by Dunn and Rajagopal [15].

In the following we will seek a velocity field of the form [13]:

$$
\mathbf{v}=\mathbf{v}(y, z, t)=v(y, z, t) \mathbf{i},
$$


where $\mathbf{i}$ denotes the unit vector along the $x$-direction of the Cartesian coordinate system $x, y$, and $z$. For these flows, the constraint of incompressibility is automatically satisfied.

Substituting (2.1)-(2.4) into the balance of linear momentum and neglecting the body forces we attain to the linear partial differential equation (cf. $[13,16])$

$$
\partial_{t} v(y, z, t)=-\frac{1}{\rho} \partial_{x} p+\left(\nu+\alpha \partial_{t}\right)\left(\partial_{y}^{2}+\partial_{z}^{2}\right) v(y, z, t)=0
$$

where $\nu=\mu / \rho$ is the kinematic viscosity of the fluid ( $\rho$ being its constant density) and $\alpha=\alpha_{1} / \rho$. Equation (2.5) with appropriate boundary and initial conditions can be solved in general by several methods. The Laplace transform can be applied to eliminate the time variable. However, the inversion procedure for obtaining the solution is not always a trivial matter. Furthermore, the solution so obtained for a second-grade fluid does not satisfy the initial condition $[17,18]$. This is due to the incompatibility between the prescribed data. Here, we will use the double Fourier sine transform.

\section{Starting flow due to an oscillating edge}

Consider an incompressible second-grade fluid at rest occupying the space of the first dial of a rectangular edge $(-\infty<x<\infty ; y, z \geq 0)$. At time $t=0^{+}$, the infinitely extended edge begins to oscillate along of $x$-axis. Owing to the shear the fluid is gradually moved. Its velocity is of the form (2.4), and the governing equation, in the absence of a pressure gradient in the $x$-direction, is

$$
\partial_{t} v(y, z, t)=\left(\nu+\alpha \partial_{t}\right)\left(\partial_{y}^{2}+\partial_{z}^{2}\right) v(y, z, t), \quad y, z, t>0 .
$$

Accordingly, the boundary and initial conditions are

$$
\begin{aligned}
& v(y, z, 0)=0, \quad y, z>0, \\
& v(0, z, t)=v(y, 0, t)=V \cos (\omega t) \quad \text { or } \quad v(0, z, t)=v(y, 0, t)=V \sin (\omega t), \quad t>0,
\end{aligned}
$$

where $\omega$ is the frequency of the velocity of the edge. The natural conditions [16]

$$
v(y, z, t), \quad \partial_{y} v(y, z, t), \quad \partial_{z} v(y, z, t) \longrightarrow 0 \quad \text { as } y^{2}+z^{2} \longrightarrow \infty, t>0
$$

have to be also satisfied.

Multiplying both sides of $(3.1)$ by $(2 / \pi) \sin (\xi y) \sin (\eta z)$, integrating with respect to $y$ and $z$ from 0 to $\infty$ and having the conditions (3.2)-(3.4) in mind, we find that [19]

$$
\begin{aligned}
& {\left[1+\alpha\left(\xi^{2}+\eta^{2}\right)\right] \partial_{t} v_{s}(\xi, \eta, t)+\nu\left(\xi^{2}+\eta^{2}\right) v_{s}(\xi, \eta, t)} \\
& =\frac{2 V}{\pi} \frac{\xi^{2}+\eta^{2}}{\xi \eta}[\nu \cos (\omega t)-\alpha \omega \sin (\omega t)], \quad \xi, \eta, t>0,
\end{aligned}
$$

respectively,

$$
\begin{aligned}
& {\left[1+\alpha\left(\xi^{2}+\eta^{2}\right)\right] \partial_{t} v_{s}(\xi, \eta, t)+\nu\left(\xi^{2}+\eta^{2}\right) v_{s}(\xi, \eta, t)} \\
& =\frac{2 V}{\pi} \frac{\xi^{2}+\eta^{2}}{\xi \eta}[\nu \sin (\omega t)+\alpha \omega \cos (\omega t)], \quad \xi, \eta, t>0,
\end{aligned}
$$


4 Solutions for motions of second-grade fluids

where the double Fourier sine transform $v_{s}(\xi, \eta, t)$ of $v(y, z, t)$ has to satisfy the initial condition

$$
v(\xi, \eta, 0)=0, \quad \xi, \eta>0 .
$$

Solving the ordinary differential equations (3.5) and (3.6) subject to the initial condition (3.7) and inverting the results by means of the Fourier's sine formula, we get for $v(y, z, t)$ the expressions (see also [19], the line 1 of Table 5)

$$
\begin{aligned}
v(y, z, t)= & V \cos (\omega t) \\
& -\frac{4 \omega^{2}}{\pi^{2}} V \cos (\omega t) \iint_{0}^{\infty} \frac{1+\alpha\left(\xi^{2}+\eta^{2}\right)}{\nu^{2}\left(\xi^{2}+\eta^{2}\right)^{2}+\omega^{2}\left[1+\alpha\left(\xi^{2}+\eta^{2}\right)\right]^{2}} \frac{\sin (y \xi)}{\xi} \frac{\sin (z \eta)}{\eta} d \xi d \eta \\
& +\frac{4 \nu \omega}{\pi^{2}} V \sin (\omega t) \iint_{0}^{\infty} \frac{\xi^{2}+\eta^{2}}{\nu^{2}\left(\xi^{2}+\eta^{2}\right)^{2}+\omega^{2}\left[1+\alpha\left(\xi^{2}+\eta^{2}\right)\right]^{2}} \frac{\sin (y \xi)}{\xi} \frac{\sin (z \eta)}{\eta} d \xi d \eta \\
& -\frac{4 V}{\pi^{2}} \iint_{0}^{\infty} \frac{\left(\xi^{2}+\eta^{2}\right)\left\{\nu^{2}\left(\xi^{2}+\eta^{2}\right)+\alpha \omega^{2}\left[1+\alpha\left(\xi^{2}+\eta^{2}\right)\right]\right\}}{\nu^{2}\left(\xi^{2}+\eta^{2}\right)^{2}+\omega^{2}\left[1+\alpha\left(\xi^{2}+\eta^{2}\right)\right]^{2}} \\
& \times \exp \left(-\frac{\nu\left(\xi^{2}+\eta^{2}\right)}{1+\alpha\left(\xi^{2}+\eta^{2}\right)} t\right) \frac{\sin (y \xi)}{\xi} \frac{\sin (z \eta)}{\eta} d \xi d \eta
\end{aligned}
$$

respectively,

$$
\begin{aligned}
v(y, z, t)= & V \sin (\omega t) \\
& -\frac{4 v \omega}{\pi^{2}} V \cos (\omega t) \iint_{0}^{\infty} \frac{\xi^{2}+\eta^{2}}{\nu^{2}\left(\xi^{2}+\eta^{2}\right)^{2}+\omega^{2}\left[1+\alpha\left(\xi^{2}+\eta^{2}\right)\right]^{2}} \frac{\sin (y \xi)}{\xi} \frac{\sin (z \eta)}{\eta} d \xi d \eta \\
& -\frac{4 \omega^{2}}{\pi^{2}} V \sin (\omega t) \iint_{0}^{\infty} \frac{1+\alpha\left(\xi^{2}+\eta^{2}\right)}{\nu^{2}\left(\xi^{2}+\eta^{2}\right)^{2}+\omega^{2}\left[1+\alpha\left(\xi^{2}+\eta^{2}\right)\right]^{2}} \frac{\sin (y \xi)}{\xi} \frac{\sin (z \eta)}{\eta} d \xi d \eta \\
& +\frac{4 \nu \omega V}{\pi^{2}} \iint_{0}^{\infty} \frac{\xi^{2}+\eta^{2}}{v^{2}\left(\xi^{2}+\eta^{2}\right)^{2}+\omega^{2}\left[1+\alpha\left(\xi^{2}+\eta^{2}\right)\right]^{2}} \\
& \times \exp \left(-\frac{v\left(\xi^{2}+\eta^{2}\right)}{1+\alpha\left(\xi^{2}+\eta^{2}\right)} t\right) \frac{\sin (y \xi)}{\xi} \frac{\sin (z \eta)}{\eta} d \xi d \eta .
\end{aligned}
$$

For large values of $t$, the starting solutions (3.8) and (3.9) reduce to the steady-state solutions which are independent of the initial condition and periodic in time. The first of 
them, for instance, can be written under the form (see the entry 1 of Table 5 from [19])

$$
\begin{aligned}
v_{s}(y, z, t)= & \frac{4 V}{\pi^{2}} \cos (\omega t) \iint_{0}^{\infty} \frac{\left(\xi^{2}+\eta^{2}\right)\left\{\nu^{2}\left(\xi^{2}+\eta^{2}\right)+\alpha \omega^{2}\left[1+\alpha\left(\xi^{2}+\eta^{2}\right)\right]\right\}}{\nu^{2}\left(\xi^{2}+\eta^{2}\right)^{2}+\omega^{2}\left[1+\alpha\left(\xi^{2}+\eta^{2}\right)\right]^{2}} \\
& \times \frac{\sin (y \xi)}{\xi} \frac{\sin (z \eta)}{\eta} d \xi d \eta \\
+ & \frac{4 \nu \omega}{\pi^{2}} \sin (\omega t) \iint_{0}^{\infty} \frac{\xi^{2}+\eta^{2}}{v^{2}\left(\xi^{2}+\eta^{2}\right)^{2}+\omega^{2}\left[1+\alpha\left(\xi^{2}+\eta^{2}\right)\right]^{2}} \frac{\sin (y \xi)}{\xi} \frac{\sin (z \eta)}{\eta} d \xi d \eta .
\end{aligned}
$$

\section{Starting flow in a duct of rectangular cross-section oscillating parallel to its length}

Let us now consider an incompressible second-grade fluid at rest in a duct of rectangular cross-section whose sides are at $y=0, y=d, z=0$, and $z=h$. At time $t=0^{+}$, the duct suddenly starts oscillating parallel to its length. By the influence of shear the fluid is gradually moved. The governing equation is again (3.1), the initial condition is (3.2), while the boundary conditions are

$$
v(0, z, t)=v(d, z, t)=v(y, 0, t)=v(y, h, t)=V \cos (\omega t), \quad t>0,
$$

or

$$
v(0, z, t)=v(d, z, t)=v(y, 0, t)=v(y, h, t)=V \sin (\omega t), \quad t>0 .
$$

The solution of this problem can be determined by means of finite double Fourier sine transforms. Thus, multiplying both sides of (3.1) by $\sin \left(\lambda_{m} y\right) \sin \left(\mu_{n} z\right)$, integrating with respect to $y$ and $z$ over $[0, d] \times[0, h]$, and taking into account (3.2) and (4.1), we find that (see $[20$, Section 13])

$\dot{v}_{m n}(t)+\frac{\nu \beta_{m n}^{2}}{1+\alpha \beta_{m n}^{2}} v_{m n}(t)=\frac{V \beta_{m n}^{2}\left[1-(-1)^{m}\right]\left[1-(-1)^{n}\right]}{\lambda_{m} \mu_{n}\left(1+\alpha \beta_{m n}^{2}\right)}[\nu \cos (\omega t)-\alpha \omega \sin (\omega t)], \quad t>0$,

where $\lambda_{m}=m \pi / d, \mu_{n}=n \pi / h, \beta_{m n}^{2}=\lambda_{m}^{2}+\mu_{n}^{2}$, and the Fourier sine transforms $v_{m n}(t)$ of $v(x, y, t)$ have to satisfy the initial conditions

$$
v_{m n}(0)=0 ; \quad m, n=1,2,3, \ldots
$$

Solving the ordinary differential equations (4.3) subject to the initial conditions (4.4) and using the Fourier inversion theorem (see [20, Section 13, equation 36]) we can write 
6 Solutions for motions of second-grade fluids

the velocity field $v(y, z, t)$ in the form

$$
\begin{aligned}
v(y, z, t)= & V \cos (\omega t) \\
& -\frac{16 \omega^{2}}{d h} V \cos (\omega t) \sum_{m, n=1}^{\infty} \frac{1+\alpha \beta_{M N}^{2}}{v^{2} \beta_{M N}^{4}+\omega^{2}\left(1+\alpha \beta_{M N}^{2}\right)^{2}} \frac{\sin \left(\lambda_{M} y\right)}{\lambda_{M}} \frac{\sin \left(\mu_{N} z\right)}{\mu_{N}} \\
& +\frac{16 \nu \omega}{d h} V \sin (\omega t) \sum_{m, n=1}^{\infty} \frac{\beta_{M N}^{2}}{v^{2} \beta_{M N}^{4}+\omega^{2}\left(1+\alpha \beta_{M N}^{2}\right)^{2}} \frac{\sin \left(\lambda_{M} y\right)}{\lambda_{M}} \frac{\sin \left(\mu_{N} z\right)}{\mu_{N}} \\
& -\frac{16 V}{d h} \sum_{m, n=1}^{\infty} \frac{\beta_{M N}^{2}\left[\nu^{2} \beta_{M N}^{2}+\alpha \omega^{2}\left(1+\alpha \beta_{M N}^{2}\right)\right]}{v^{2} \beta_{M N}^{4}+\omega^{2}\left(1+\alpha \beta_{M N}^{2}\right)^{2}} \\
& \times \exp \left(-\frac{v \beta_{M N}^{2}}{1+\alpha \beta_{M N}^{2}} t\right) \frac{\sin \left(\lambda_{M} y\right)}{\lambda_{M}} \frac{\sin \left(\mu_{N} z\right)}{\mu_{N}},
\end{aligned}
$$

where $M=2 m-1$ and $N=2 n-1$.

The starting solution corresponding to the boundary conditions (4.2) is

$$
\begin{aligned}
v(y, z, t)= & V \sin (\omega t) \\
& -\frac{16 \nu \omega}{d h} V \cos (\omega t) \sum_{m, n=1}^{\infty} \frac{\beta_{M N}^{2}}{\nu^{2} \beta_{M N}^{4}+\omega^{2}\left(1+\alpha \beta_{M N}^{2}\right)^{2}} \frac{\sin \left(\lambda_{M} y\right)}{\lambda_{M}} \frac{\sin \left(\mu_{N} z\right)}{\mu_{N}} \\
& -\frac{16 \omega^{2}}{d h} V \sin (\omega t) \sum_{m, n=1}^{\infty} \frac{1+\alpha \beta_{M N}^{2}}{\nu^{2} \beta_{M N}^{4}+\omega^{2}\left(1+\alpha \beta_{M N}^{2}\right)^{2}} \frac{\sin \left(\lambda_{M} y\right)}{\lambda_{M}} \frac{\sin \left(\mu_{N} z\right)}{\mu_{N}} \\
& \left.+\frac{16 \nu \omega V}{d h} \sum_{m, n=1}^{\infty} \frac{\beta_{M N}^{2} \beta_{M N}^{4}+\omega^{2}\left(1+\alpha \beta_{M N}^{2}\right)^{2}}{\nu \beta_{M N}^{2}} t\right) \frac{\sin \left(\lambda_{M} y\right)}{\lambda_{M}} \frac{\sin \left(\mu_{N} z\right)}{\mu_{N}} . \\
& \times \exp \left(-\frac{\alpha \beta_{M N}^{2}}{1+}\right.
\end{aligned}
$$

\section{Starting flow due to an oscillating pressure gradient}

Let us now assume that at time $t=0^{+}$a pressure gradient of the form

$$
\partial_{x} p=-\rho Q \cos (\omega t) \quad \text { or } \quad \partial_{x} p=-\rho Q \sin (\omega t)
$$


acts on the fluid laid in a duct of rectangular cross-section. The governing equation is given by (2.5), the initial condition is (3.2), while the boundary conditions are

$$
v(0, z, t)=v(d, z, t)=v(y, 0, t)=v(y, h, t)=0, \quad t>0
$$

The exact solutions corresponding to these last two flows have the forms

$$
\begin{aligned}
v(y, z, t)= & \frac{16 Q}{d h} \sum_{m, n=1}^{\infty} \frac{\nu \beta_{M N}^{2} \cos (\omega t)+\omega\left(1+\alpha \beta_{M N}^{2}\right) \sin (\omega t)}{\nu^{2} \beta_{M N}^{4}+\omega^{2}\left(1+\alpha \beta_{M N}^{2}\right)^{2}} \frac{\sin \left(\lambda_{M} y\right)}{\lambda_{M}} \frac{\sin \left(\mu_{N} z\right)}{\mu_{N}} \\
& -\frac{16 \nu Q}{d h} \sum_{m, n=1}^{\infty} \frac{\beta_{M N}^{2}}{\nu^{2} \beta_{M N}^{4}+\omega^{2}\left(1+\alpha \beta_{M N}^{2}\right)^{2}} \exp \left(-\frac{v \beta_{M N}^{2}}{1+\alpha \beta_{M N}^{2}} t\right) \frac{\sin \left(\lambda_{M} y\right)}{\lambda_{M}} \frac{\sin \left(\mu_{N} z\right)}{\mu_{N}}
\end{aligned}
$$

respectively,

$$
\begin{aligned}
v(y, z, t)= & \frac{16 Q}{d h} \sum_{m, n=1}^{\infty} \frac{\nu \beta_{M N}^{2} \sin (\omega t)-\omega\left(1+\alpha \beta_{M N}^{2}\right) \cos (\omega t)}{\nu^{2} \beta_{M N}^{4}+\omega^{2}\left(1+\alpha \beta_{M N}^{2}\right)^{2}} \frac{\sin \left(\lambda_{M} y\right)}{\lambda_{M}} \frac{\sin \left(\mu_{N} z\right)}{\mu_{N}} \\
& +\frac{16 \omega Q}{d h} \sum_{m, n=1}^{\infty} \frac{1+\alpha \beta_{M N}^{2}}{\nu^{2} \beta_{M N}^{4}+\omega^{2}\left(1+\alpha \beta_{M N}^{2}\right)^{2}} \\
& \times \exp \left(-\frac{\nu \beta_{M N}^{2}}{1+\alpha \beta_{M N}^{2}} t\right) \frac{\sin \left(\lambda_{M} y\right)}{\lambda_{M}} \frac{\sin \left(\mu_{N} z\right)}{\mu_{N}} .
\end{aligned}
$$

\section{Conclusions}

In this paper, the velocity fields corresponding to the motions of a second-grade fluid due to the sine and cosine oscillations of an infinite edge and of an infinite channel of rectangular cross-section as well as those produced by an oscillating pressure gradient have been determined by means of the Fourier sine transforms. The solutions that have been obtained, depending on the initial and boundary conditions, are presented as sum of the steady-state and transient solutions. They describe the flows for small and large times. For large values of time $t$, when the transients disappear, all motions are described by the corresponding steady-state solutions, which are periodic in time and independent of the initial condition.

Straightforward computations show that $v(y, z, t)$, given by (3.8), (3.9), (4.5), (4.6), (5.3), and (5.4), satisfies both the associate partial differential equations (2.5) and (3.1) and all imposed initial and boundary conditions, the differentiations under integrals or term by term in sums being clearly permissible. In the special case when $\alpha_{1} \rightarrow 0$, corresponding to a Reiner-Rivlin fluid, all solutions take the simpler forms. Equation (3.8), for 
example, takes the form (the last term giving the transient solution)

$$
\begin{aligned}
v(y, z, t)= & V \cos (\omega t) \\
& -\frac{4}{\pi^{2}}\left(\frac{\omega}{\nu}\right)^{2} V \cos (\omega t) \iint_{0}^{\infty} \frac{1}{\left(\xi^{2}+\eta^{2}\right)^{2}+(\omega / \nu)^{2}} \frac{\sin (y \xi)}{\xi} \frac{\sin (z \eta)}{\eta} d \xi d \eta \\
& +\frac{4}{\pi^{2}}\left(\frac{\omega}{\nu}\right)^{2} V \sin (\omega t) \iint_{0}^{\infty} \frac{\xi^{2}+\eta^{2}}{\left(\xi^{2}+\eta^{2}\right)^{2}+(\omega / \nu)^{2}} \frac{\sin (y \xi)}{\xi} \frac{\sin (z \eta)}{\eta} d \xi d \eta \\
& -\frac{4 V}{\pi^{2}} \iint_{0}^{\infty} \frac{\left(\xi^{2}+\eta^{2}\right)^{2}}{\left(\xi^{2}+\eta^{2}\right)^{2}+(\omega / \nu)^{2}} \exp \left[-\nu\left(\xi^{2}+\eta^{2}\right) t\right] \frac{\sin (y \xi)}{\xi} \frac{\sin (z \eta)}{\eta} d \xi d \eta,
\end{aligned}
$$

which is identical to that for a Navier-Stokes fluid. However, the surface tractions that must be applied in order to produce the motion will vary according to the values of $\alpha_{2}$.

\section{References}

[1] H. Schlichting, Boundary Layer Theory, McGraw-Hill, New York, 6th edition, 1968.

[2] M. E. Erdoğan, "A note on an unsteady flow of a viscous fluid due to an oscillating plane wall," International Journal of Non-Linear Mechanics, vol. 35, no. 1, pp. 1-6, 2000.

[3] C. Fetecau and C. Fetecau, "Starting solutions for some unsteady unidirectional flows of a second grade fluid," International Journal of Engineering Science, vol. 43, no. 10, pp. 781-789, 2005.

[4] K. R. Rajagopal, "A note on unsteady unidirectional flows of a non-Newtonian fluid," International Journal of Non-Linear Mechanics, vol. 17, no. 5-6, pp. 369-373, 1982.

[5] K. R. Rajagopal, "Longitudinal and torsional oscillations of a rod in a non-Newtonian fluid," Acta Mechanica, vol. 49, no. 3-4, pp. 281-285, 1983.

[6] K. R. Rajagopal and R. K. Bhatnagar, "Exact solutions for some simple flows of an Oldroyd-B fluid," Acta Mechanica, vol. 113, no. 1-4, pp. 233-239, 1995.

[7] T. Hayat, S. Asghar, and A. M. Siddiqui, "Periodic unsteady flows of a non-Newtonian fluid," Acta Mechanica, vol. 131, no. 3-4, pp. 169-175, 1998.

[8] T. Hayat, S. Asghar, and A. M. Siddiqui, "Some unsteady unidirectional flows of a nonNewtonian fluid," International Journal of Engineering Science, vol. 38, no. 3, pp. 337-346, 2000.

[9] T. Hayat, A. M. Siddiqui, and S. Asghar, "Some simple flows of an Oldroyd-B fluid," International Journal of Engineering Science, vol. 39, no. 2, pp. 135-137, 2001.

[10] A. M. Siddiqui, T. Hayat, and S. Asghar, "Periodic flows of a non-Newtonian fluid between two parallel plates," International Journal of Non-Linear Mechanics, vol. 34, no. 5, pp. 895-899, 1999.

[11] S. Asghar, T. Hayat, and A. M. Siddiqui, "Moving boundary in a non-Newtonian fluid," International Journal of Non-Linear Mechanics, vol. 37, no. 1, pp. 75-80, 2002.

[12] C. Fetecau and C. Fetecau, "Starting solutions for the motion of a second grade fluid due to longitudinal and torsional oscillations of a circular cylinder," International Journal of Engineering Science, vol. 44, no. 11-12, pp. 788-796, 2006.

[13] M. E. Erdoğan and C. E. İmrak, "Effects of the side walls on the unsteady flow of a second-grade fluid in a duct of uniform cross-section," International Journal of Non-Linear Mechanics, vol. 39, no. 8, pp. 1379-1384, 2004. 
[14] J. E. Dunn and R. L. Fosdick, "Thermodynamics, stability, and boundedness of fluids of complexity 2 and fluids of second grade," Archive for Rational Mechanics and Analysis, vol. 56, no. 3, pp. 191-252, 1974.

[15] J. E. Dunn and K. R. Rajagopal, "Fluids of differential type: critical review and thermodynamic analysis," International Journal of Engineering Science, vol. 33, no. 5, pp. 689-729, 1995.

[16] C. Fetecau and C. Fetecau, "The Rayleigh-Stokes problem for heated second grade fluids," International Journal of Non-Linear Mechanics, vol. 37, no. 6, pp. 1011-1015, 2002.

[17] R. Bandelli, K. R. Rajagopal, and G. P. Galdi, "On some unsteady motions of fluids of second grade," Archives of Mechanics, vol. 47, no. 4, pp. 661-676, 1995.

[18] R. Bandelli and K. R. Rajagopal, "Start-up flows of second grade fluids in domains with one finite dimension," International Journal of Non-Linear Mechanics, vol. 30, no. 6, pp. 817-839, 1995.

[19] I. N. Sneddon, "Functional analysis," in Encyclopedia of Physics, Vol. ll, pp. 198-348, Springer, Berlin, 1955.

[20] I. N. Sneddon, Fourier Transforms, McGraw-Hill, New York, 1951.

C. Fetecau: Department of Mathematics, Technical University of Iasi, 6600 Iasi, Romania E-mail address: fetecau@math.tuiasi.ro

Corina Fetecau: Department of Theoretical Mechanics, Technical University of Iasi, 6600 Iasi, Romania

E-mail address: cfetecau@ontario.tcm.tuiasi.ro 


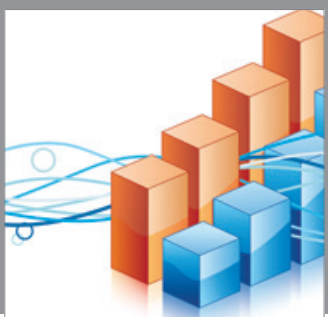

Advances in

Operations Research

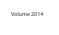

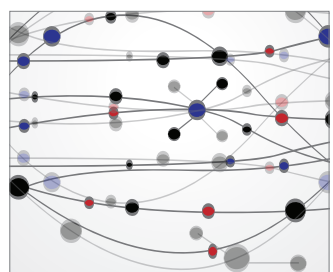

\section{The Scientific} World Journal
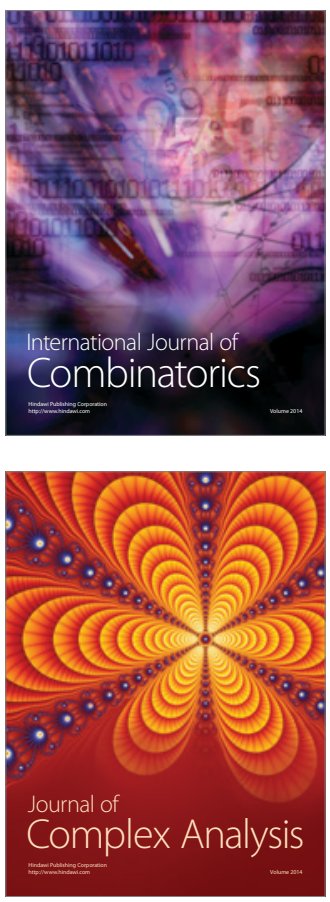

International Journal of

Mathematics and

Mathematical

Sciences
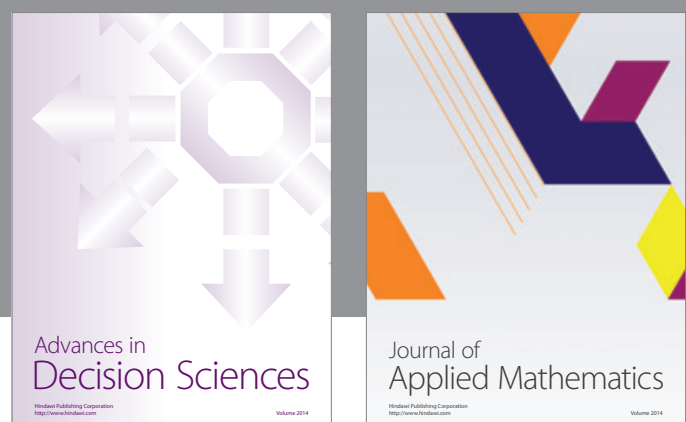

Journal of

Applied Mathematics
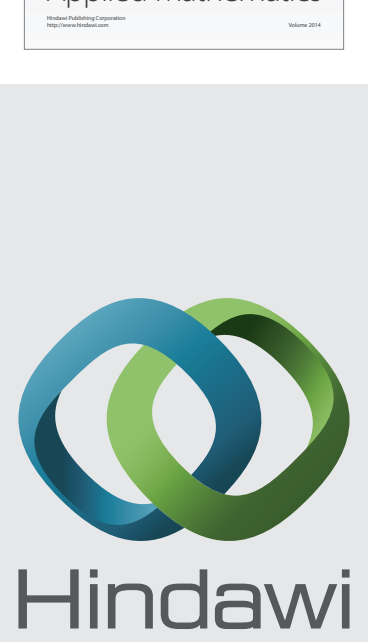

Submit your manuscripts at http://www.hindawi.com
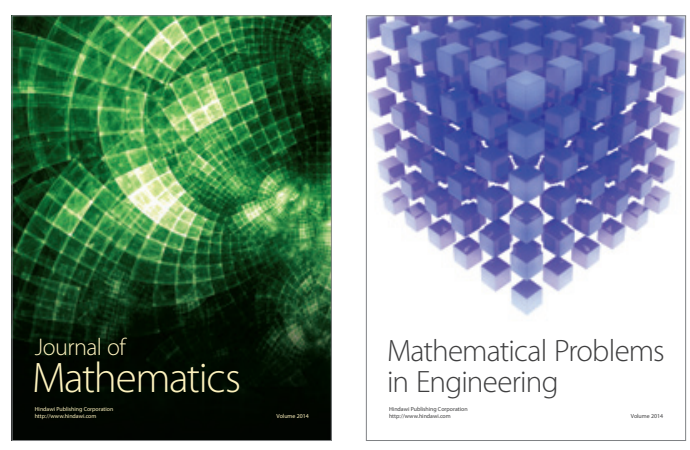

Mathematical Problems in Engineering
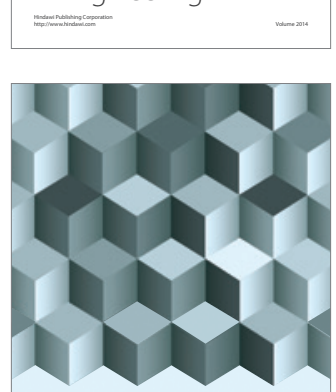

Journal of

Function Spaces
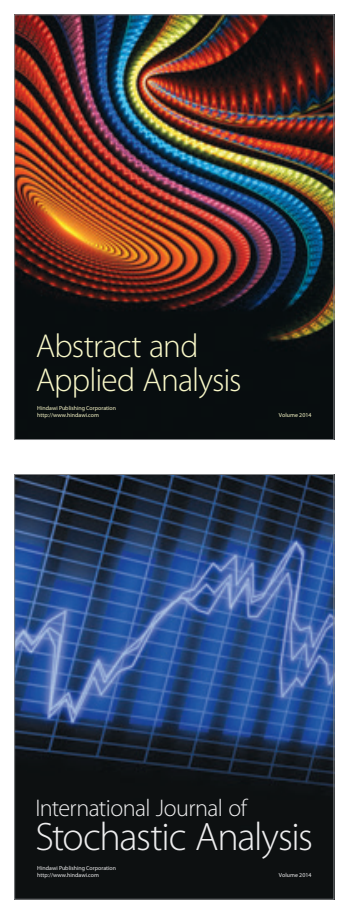

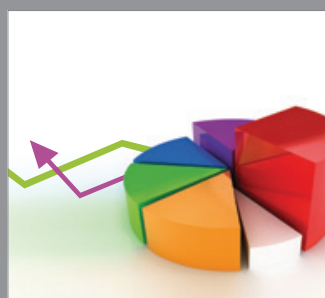

ournal of

Probability and Statistics

Promensencen
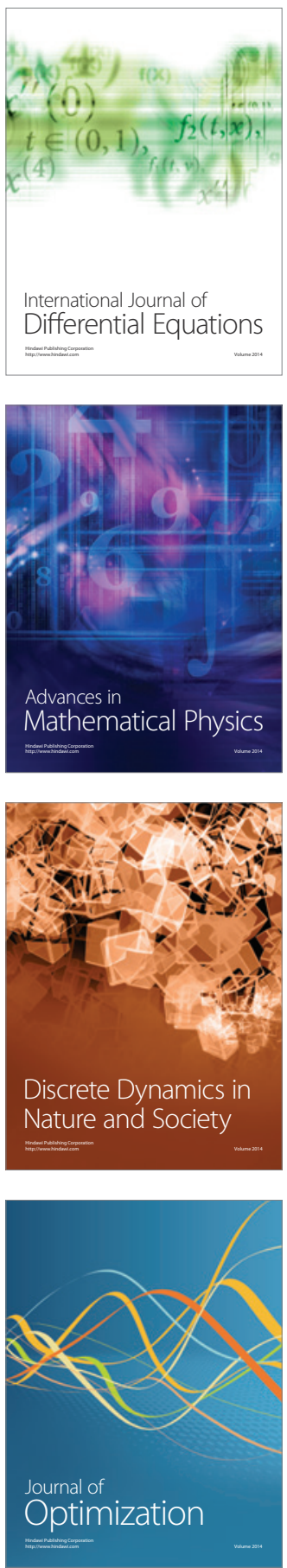